\title{
Room-temperature, high-efficiency conversion of Mott- Wannier excitons to Frenkel excitons in hybrid semiconductor quantum dot/polymer composites
}

\author{
Sedat Nizamoglu ${ }^{1}$, Xiao Wei Sun ${ }^{2}$ and Hilmi Volkan Demir ${ }^{1,2}$ \\ ${ }^{I}$ Department of Electrical and Electronics Engineering, Department of Physics, and \\ UNAM - Institute of Materials Science and Nanotechnology, Bilkent University, Ankara, Turkey 06800 \\ ${ }^{2}$ School of Electrical and Electronic Engineering, Microelectronics Division; School of Physical and Mathematical Sciences, Physics and \\ Applied Physics Division, Nanyang Technological University, Singapore 639798 \\ Email:volkan@bilkent.edu.tr and hvdemir@ntu.edu.sg
}

\begin{abstract}
Efficient conversion from Mott-Wannier to Frenkel excitons at room temperature is observed in hybrid inorganic/organic composites of $\mathrm{CdSe} / \mathrm{ZnS}$ core/shell heteronanocrystals in MDMO-PPV homopolymers at a rate of $0.2628 \mathrm{~ns}^{-1}$ with an efficiency of $80.9 \%$.

(C)2011 Optical Society of America

OCIS codes: (260.2160) Energy transfer; (160.4236) Nanomaterials; (160.4760) Optical properties
\end{abstract}

The Mott-Wannier (MW) and Frenkel (FR) excitons are the primary Coulomb-correlated light generation tools in dielectric media. In disordered inorganic/organic composite systems, incoherent coupling via Förster-type nonradiative energy transfer (ET) enables conversion of MW excitons to FR excitons [1-2]. In a recent study, Blumentengel et al. investigated this excitonic conversion process [1]. However, this was observable only up to a maximum temperature of $100 \mathrm{~K}$ with a moderate energy transfer efficiency level of $50 \%$. In that previous work, the energy transfer was terminated above $100 \mathrm{~K}$ because the excitons were reported to dissociate. To make an efficient system, though, the energy transfer efficiency needs to be higher than $50 \%$ (i.e., most of the excitons in inorganic substance should migrate to the organic domain). Also this migration is desired to be achieved at room temperature for potential optoelectronic applications. But, such efficient exciton conversion has not been shown at room temperature to date.

In this work, we study and demonstrate room-temperature, high-efficiency conversion from Mott-Wannier to Frenkel excitons through nonradiative ET. At room temperature, an ET rate of $0.1479 \mathrm{~ns}^{-1}$, together with an efficiency of $80.9 \%$, was realized. ET was revealed with the simultaneous observation of both decreased donor lifetime and increased acceptor lifetime. Furthermore, the experimental and analytical ET efficiencies were found to be in excellent agreement [3]. For that, cyan-emitting CdSe/ZnS core/shell heteronanocrystals (NCs) serving as the donors and orange-emitting poly[2-methoxy-5-(3,7-dimethyl-octyloxy)-1,4-phenylenevinylene] (MDMO-PPV) homopolymers serving as the acceptors were investigated. These were carefully chosen to favorably exhibit strong spectral overlap between the emission peak of the heteronanocrystals and the absorption peak of the homopolymers. The homopolymer concentration was then controlled with great care; the polymer concentrations were set approximately to $1.5 \times 10^{17}, 2.9 \times 10^{17}, 4.4 \times 10^{17}$, and $5.3 \times 10^{17} \mathrm{~cm}^{-3}$ in Samples $1,2,3$ and 4, respectively, and the heteronanocrystal concentration was kept around $10^{18} \mathrm{~cm}^{-3}$ in all of the samples.
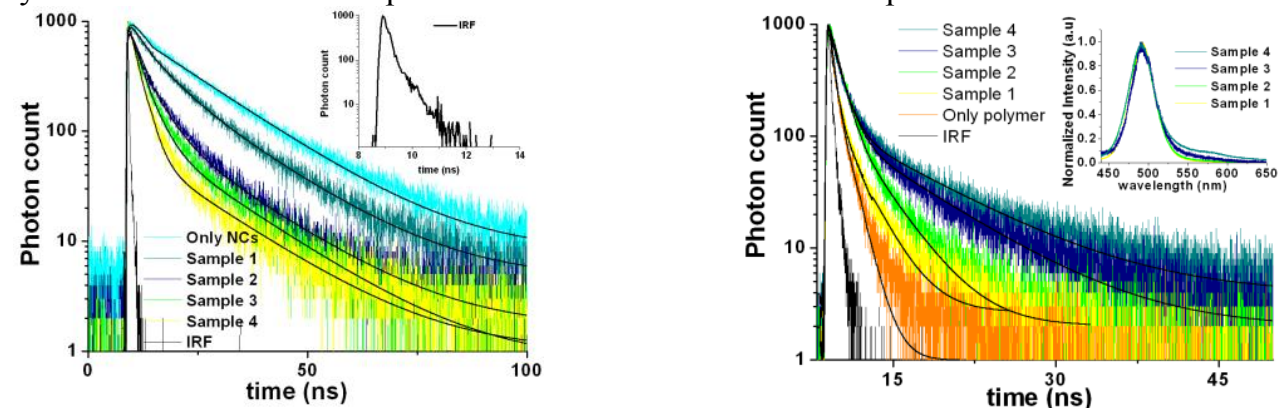

Figure. 1 Time-resolved spectroscopy measurements of the hybrid composite system consisting of heteronanocrystals and homopolymers together (Samples 1-4) at the peak heteronanocrystal emission wavelength, $495 \mathrm{~nm}$ (left) and at the peak homopolymer emission wavelength, 585 $\mathrm{nm}$ (right), along with the laser impulse response (given in the left inset) and the steady-state photoluminescence spectra from Samples 1-4 (given in the right inset), all at room temperature.

Fig. 1 shows the time-resolved spectroscopy of our hybrid homopolymer-heteronanocrystal composites along with that of NC-only solids on quartz substrates at the nanocrystal donor emission, $495 \mathrm{~nm}$ (left). For the NC-only sample, we fit the photoluminescence decay using a single lifetime component of $16.163 \mathrm{~ns}$ (i.e., $\Gamma_{\mathrm{NC}}=0.0619 \mathrm{~ns}^{-1}$ ), which is the recombination lifetime of the nanocrystals (around tens of nanoseconds) at room temperature. In 


\section{QTuL6.pdf}

Sample 1, when NCs are blended in MDMO-PPV homopolymers, the photoluminescence of NCs starts to quench due to the exciton migration from NCs to polymers. Thus, in addition to the interband recombination process, an extra decay component appears as a result of the nonradiative energy transfer. For Sample 1, this additional lifetime component is $7.00 \mathrm{~ns}$ (i.e., $\Gamma_{\text {sample1 }}=0.1429 \mathrm{~ns}^{-1}$ ), which is faster than the competing recombination lifetime of heteronanocrystals alone. We thus determined this nonradiative ET rate from the difference of the rates, $\Gamma_{\mathrm{ET}}=$ $\Gamma_{\text {sample } 1}-\Gamma_{\mathrm{NC}}=0.1429-0.0619=0.0810 \mathrm{~ns}^{-1}$. The resulting efficiency for Sample $1\left(\eta=\Gamma_{\mathrm{ET}} / \Gamma_{\text {sample1 }}\right)$ is then $56.6 \%$ at room temperature. As we further increase the acceptor concentration in Samples 2, 3 and 4, the resultant quenching increases correspondingly. For these cases, the respective ET rates are $0.1085,0.1791$ and $0.2628 \mathrm{~ns}^{-1}$, again as a result of the increased ET from heteronanocrystals to homopolymers. These correspond to the roomtemperature efficiency levels of $63.6 \%, 74.3 \%$, and finally $80.9 \%$, respectively. For all samples, this means that more than half of the excitation energy of heteronanocrystals is transferred to homopolymers at room temperature.

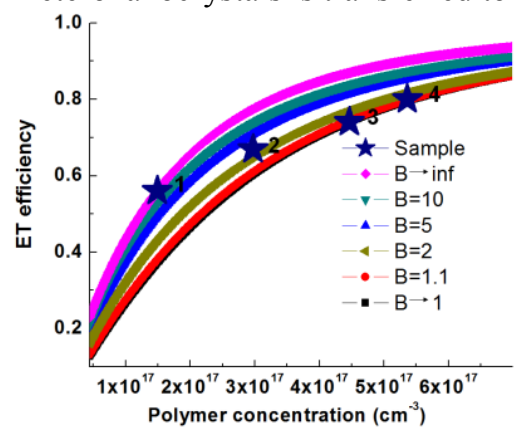

Figure 2. Calculated ET efficiencies based on the analytical model using measured ET efficiencies and different B values.

A possible question still remains at this point: whether the energy is transferred to homopolymers or to the surface traps due to the defect states of nanocrystals, despite the observation of quenching in heteronanocrystals. To address this question, we also investigated the decay behavior of the homopolymers as shown in Fig. 1 (right). In the control group, the lifetime decay of the MDMO-PPV homopolymers can be represented as biexponentials with $\tau_{1}=$ $0.228 \mathrm{~ns}$ and $\tau_{2}=1.079 \mathrm{~ns}$ because of their Frenkel-type excitonic behavior. As the acceptor homopolymer concentration increases in the composite, their lifetime is observed to increase, which is opposite to the decreasing lifetime of donor heteronanocrystals. Furthermore, in our hybrid system, this lifetime modification cannot be due to the charge transfer (i.e., Dexter-type transfer) because ZnS barriers (of about three monolayers) in the core/shell heteronanocrystals provide full electronic isolation and prevent tunneling of the electron and hole wavefunctions. In the inset of Fig. 1 (right), the steady-state luminescence of the heteronanocrystal-homopolymer composite is presented. From Sample 1 to 4, the emission above the wavelength $550 \mathrm{~nm}$ coming from the homopolymer is increasing, which is consistent with the decreasing donor lifetime and increasing acceptor lifetime. Thus, we can undoubtedly conclude that this is the result of nonradiative energy transfer from heteronanocrystals to homopolymers.

To investigate ET efficiency $(\eta)$ in our hybrid system, we derived Eq. (1) based on quenching of donors as a function of their concentration, where $C$ is $4 \pi r_{F}^{3}(Q)^{-1 / 2}, r_{F}$ is the Förster radius for the heteronanocrystalhomopolymer interaction, $\mathrm{q}$ is the homopolymer concentration, $\mathrm{Q}$ is the quantum efficiency of the heteronanocrystal and B is the parameter showing the nonradiative ET between heteronanocrystals (i.e., if there is no ET among heteronanocrystals, then $\mathrm{B}=1$ and if ET is strong between heteronanocrystals, then $\mathrm{B} \rightarrow \infty$ ). As shown in Fig. 2 as the polymer concentration increases, the ET efficiency converges to the case of $\mathrm{B}=1$ because the intradot energy transfer becomes weaker (due to the increased polymer concentration). It is observed that the experimental results are consistent with the calculated ET efficiencies, confirming the measured efficiencies and the analytical model.

$$
\eta=1-\frac{1}{B^{2}-1}\left(\frac{B^{2}+(C q)^{2}}{1+(C q)^{2}} \exp \left\{C q \pi\left(\frac{1}{B}-1\right)+2 C q\left[\arctan (C q)-\frac{\arctan (C q / B)}{B}\right]\right\}-1\right)
$$

To summarize, efficient conversion from Mott-Wannier to Frenkel excitons was demonstrated at room temperature by nonradiative ET using a hybrid composite system composed of heteronanocrystals and homopolymers. This holds great promise for future hybrid solid state devices.

We acknowledge financial support NRF-RF-2009-09, ESF-EURYI, EC FP7 N4E-NoE, TUBA- GEBIP, TUBITAK-BIDEB and TUBITAK 107E088, 109E002, 109E004, and 110E010.

References

[1] S. Blumstengel et. al., Phys. Rev. Lett., 97, 237401 (2006).

[2] V. M. Agranovich et. al., J. Phys. Condens. Matter. 10, 9369 (1998).

[3] S. Nizamoglu et. al., Appl. Phys. Lett., (in press). 